\title{
V032 PRESTACK DEPTH MIGRATION BY 3D TOMOGRAPHIC INVERSION OF REFLECTED AND REFRACTED ARRIVALS
}

A. VESNAVER' ${ }^{1}$, H. BIAOLONG ${ }^{2}$, G. BÖHM ${ }^{1}$, G. MADRUSSANI ${ }^{1}$, S. PETERSEN ${ }^{3}$ and G. ROSSI' 'OGS, PO Box 2011 Opicina, 34016 Trieste, Italy

Reflection tomography produces reliable macro-models in depth for the velocity field of seismic waves and other geophysical properties, when complex 3-D structures are present. The well-known inversion ambiguities due to the null space can be avoided by fitting the grid to the distribution of the available ray paths. While reflected arrivals provide mainly the reflector depth and the vertical velocity variations, the refracted arrivals bear important information about the lateral velocity changes. This is, because the ray paths of the first ones are nearly vertical; viceversa, the second ones contain parts travelling nearly horizontally along the layer interfaces. So the joint inversion of reflected and refracted arrivals increases the resolution or the stability of the obtained models.

When a good macro-model is computed, we get high resolution images by the pre-stack depth migration of seismic traces. We applied these techniques to a 3-D real data set acquired at the North Sea.

Figure 1 shows the velocity field obtained by conventional and continuous velocity spectra, converted in depth by the Dix formula. We notice instabilities in the lower part due to inhomogeneities in the overburden. The estimate provided by 3-D reflection tomography (Figure 2) is much more stable.

Relevant differences occur when using these velocity fields for the 2-D pre-stack depth migration of a selected profile. In the image obtained from stacking velocity (Figure 3), we do not resolve satisfactorily some high impedance channels at a depth of about $1.4 \mathrm{~km}$, and the strong reflectors at depths larger than $2 \mathrm{~km}$ appear strongly undulated. The migrated section provided by reflection tomography (Figure 4 ) is much better resolved and stable.

The joint use of reflected and refracted arrivals is providing encouraging results: the obtained velocity fields are smoother than those estimated by reflected arrivals only. Several tests using synthetic 3-D data sets proved that significant improvements are so achieved. 


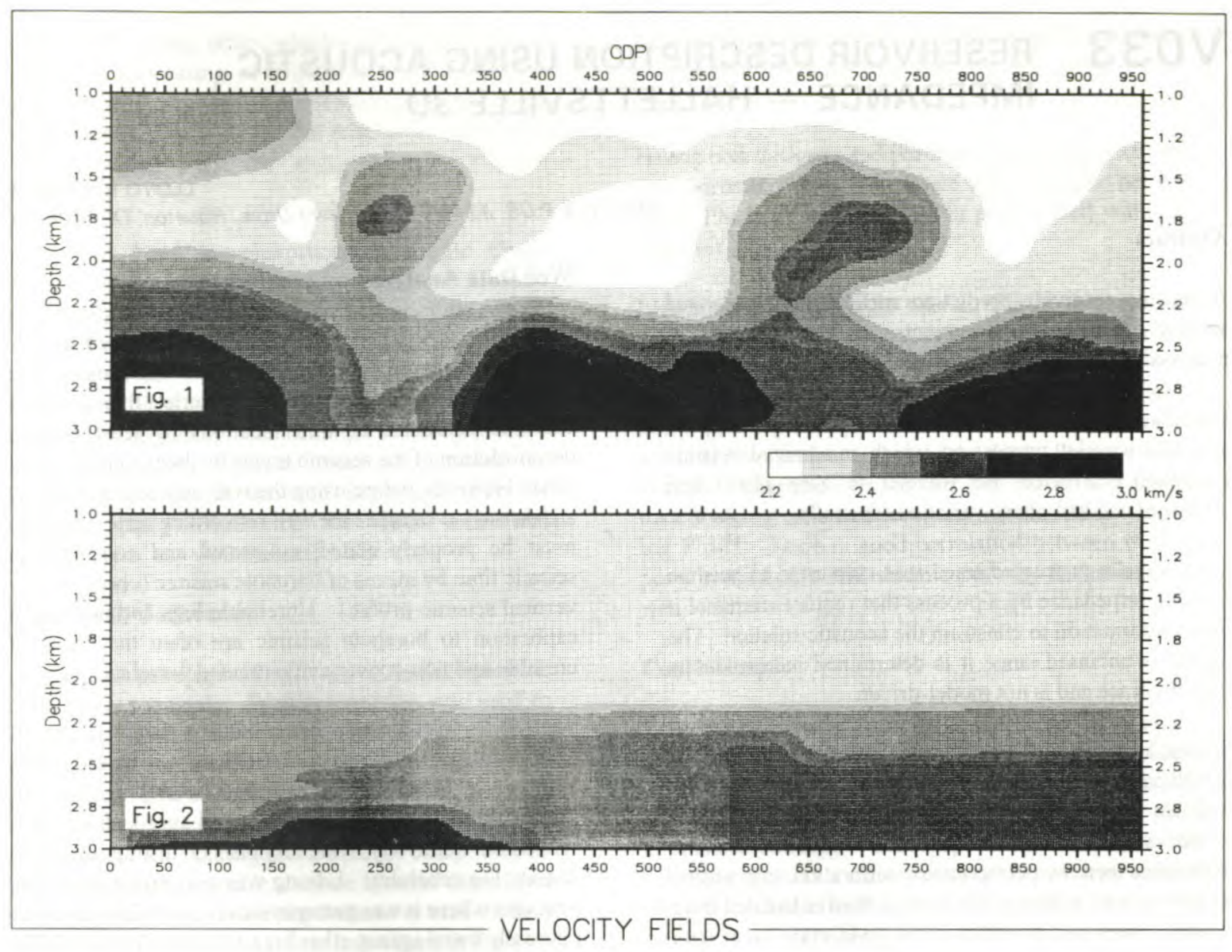

CDP:

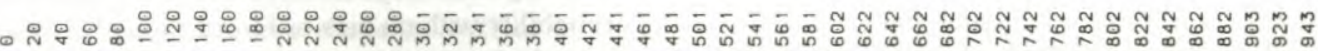
(1) CDP:

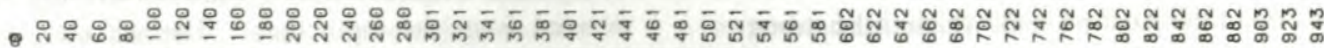
$5=1,15=5$

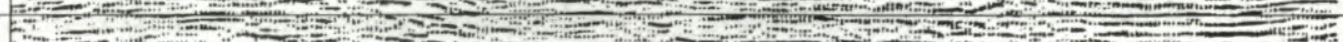

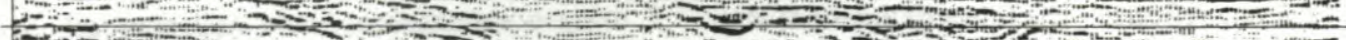

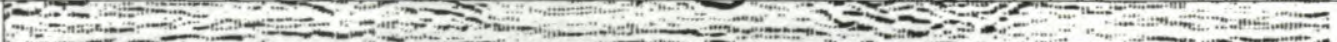

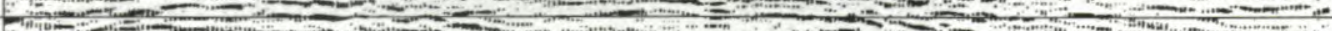
5. -

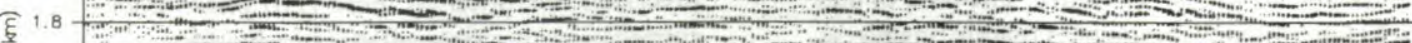

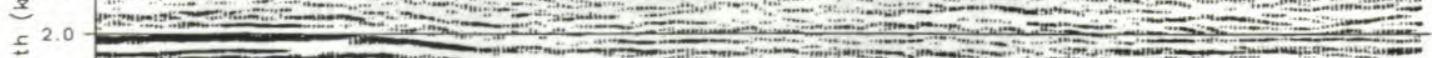

จั 2.2 -

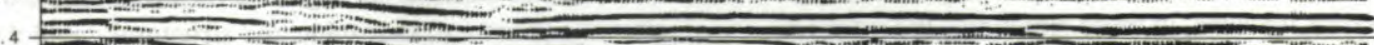

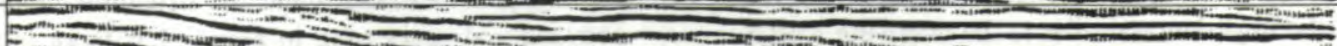

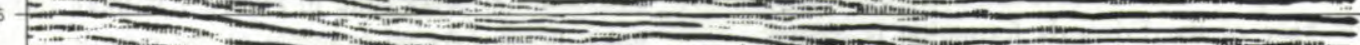

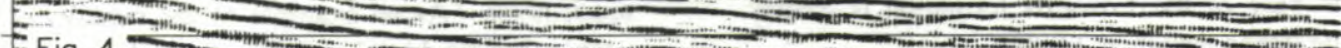
Fig. $4=0$ 